G65(P)

\section{RISK OF INVASIVE PNEUMOCOCCAL DISEASE IN CHILDREN WITH SICKLE CELL DISEASE IN THE ERA OF PNEUMOCOCCAL CONJUGATE VACCINES: A SYSTEMATIC REVIEW OF THE LITERATURE}

${ }^{1} \mathrm{G}$ Oligbu, ${ }^{2} \mathrm{~L}$ Pay, ${ }^{2} \mathrm{M}$ Fallaha, ${ }^{3} \mathrm{O}$ Oligbu, ${ }^{4} \mathrm{U}$ Nwaomu. ${ }^{1}$ Paediatric Infectious Diseases Research Group, St George's University of London, UK; ${ }^{2}$ Department of Paediatrics, Imperial College London, UK; ${ }^{3}$ Accident and Emergency Department, Queen Elizabeth Hospital, Woolwich, London, UK; ${ }^{4}$ Department of Paediatrics, Royal Derby Hospital, Derby, UK

\subsection{6/archdischild-2018-rcpch.63}

Introduction Invasive pneumococcal disease (IPD) is the leading cause of morbidity and mortality in children with sickle cell disease (SCD). 7-valent pneumococcal conjugate vaccine (PCV7) was first introduced in 2000 in US and at various times in other countries. It was replaced by 13 -valent pneumococcal conjugate vaccines (PCV13) in 2010. This PCV are highly effective in preventing IPD in children with SCD. The risk of IPD has not been systematically assessed in children with SCD since the introduction of PCV.

Methods We undertook a systematic review of the English literature published from 2000 to October 2017 to evaluate the risk factors, serotype distribution, clinical presentation and outcomes of IPD in children with sickle cell disease. Data sources included MEDLINE, EMBASE Cochrane library, and references within identified articles.

Results We identified 475 potential studies, of which 66 were duplicates. A further 299 were excluded on the basis of title and abstracts and another 94 studies did not meet eligibility criteria on full-text screening. We included 16 publications involving 11383 children less than 21 years-olds with SCD. A total of $161(1.4 \%)$ IPD was identified: 147 homozygote for haemoglobin S (HbSS), 10 double heterozygote for haemoglobin S and C (HbSC) and 4 others. Among the nine studies reporting clinical presentation, septicaemia was the commonest $(n=69 / 108 ; 63.8 \%)$ followed by pneumonia $(n=20 / 108$; $18.5 \%)$. The serotypes associated with IPD in SCD were mainly non-PCV13 ( $\mathrm{n}=117 / 133 ; 88 \%)$, of which more than a quarter was due to serogroup $15 \mathrm{~A} / \mathrm{B} / \mathrm{C} \quad(\mathrm{n}=34 / 117 ; 29 \%$,). Serotype 23 F $(n=6 / 16,37.5 \%)$ and 7 F $(n=4 / 16 ; 25 \%)$ were the main vaccine serotypes. Majority were sensitive to penicillin and ceftriaxone. The crude case fatality rate was $11.2 \%$ $(\mathrm{n}=18 / 161)$.

Conclusions and clinical implications This report demonstrates the effectiveness of conjugate vaccines to reduce the rate of IPD in children with SCD. However, these children remain at increased risk and are also more likely to die of their infection compared to their peers without SCD. Most IPD cases are now due to serotypes not covered by PCV13. Therefore better prevention strategies are needed to reduce the overall burden IPD in children with SCD.

\section{G66(P) REDUCED RATES OF JUVENILE ONSET RECURRENT RESPIRATORY PAPILLOMATOSIS IN AUSTRALIA AFTER IMPLEMENTATION OF A NATIONAL HPV VACCINATION PROGRAM}

1) Brotherton, 2,3Y Zurynski, ${ }^{4} \mathrm{~A}$ Cheng, ${ }^{5} \mathrm{SM}$ Garland, ${ }^{3,4,6} \mathrm{R}$ Booy, ${ }^{2,3,4} \mathrm{EJ}$ Elliott, ${ }^{7}$ D Novakovic. 'National HPV Vaccination Program Register, VCS, East Melbourne, Victoria, Australia; ${ }^{2}$ Australian Paediatric Surveillance Unit, Kids Research Institute, Sydney, Australia; ${ }^{3}$ Discipline of Child and Adolescent Health, The University of Sydney, Sydney, Australia; ${ }^{4}$ Sydney Children's Hospitals Network, the Children's Hospital Westmead, Sydney, Australia; ${ }^{5} 12$ Royal Women's Hospital, Department of Microbiology and Infectious Diseases, Parkville, and Murdoch Childrens Research Institute, Infection and Immunity Theme, Melbourne, Australia; ${ }^{6}$ National Centre for Immunisation Research and Surveillance, Kids Research Institute, Sydney, Australia; 'University of Sydney Medical School, University of Sydney, Sydney, Australia

\subsection{6/archdischild-2018-rcpch.64}

Aims Juvenile onset Recurrent Respiratory Papillomatosis (JoRRP) is a rare chronic disease caused by human papillomavirus (HPV) types 6 and 11. Children with RRP require multiple surgical interventions. Tracheostomy may be needed and sometimes the disease is fatal. These infections are now preventable through HPV vaccination. Following an extensive quadrivalent HPV vaccine catch-up program for females aged 12-26 years in 2007-2009, in Australia, we aimed to monitor the changes in incidence and demographics of JoRRP over time.

Methods The Australian Paediatric Surveillance Unit (APSU) conducted national surveillance for JRRoP using its well established reporting system. In addition to the $\sim 1450$ paediatricians who report to the APSU each month, paediatric otorhinolaryngologists were also enrolled in the APSU and offered HPV typing. We report findings for the five-year period to end 2016.

Results The average annual incidence rate was 0.0715 per 100000 children aged $<16$ years. The largest number of cases was reported in the first year, with decreasing annual frequency thereafter. The rate declined significantly from 0.163 per 100000 in 2012 to 0.024 per 100000 in 2016 $(\mathrm{p}=0.034)$. Among the 15 incident cases $60 \%$ male, $60 \%$ were first born children and 13 (87\%) were born vaginally. None of the mothers of these children had received the HPV vaccine before pregnancy, and $3(20 \%)$ of the mothers had a history of genital warts. Seven genotyped cases were positive for HPV including 4 that were HPV6 positive and 3 that were HPV11 positive.

Conclusion To our knowledge this is the first report internationally documenting decline in JoRRP incidence in children following a quadrivalent HPV vaccination program. 